\title{
Survey and Documentation of Brackish Water Algal Diversity from East Coast Region of Odisha, India
}

\author{
Himansu Sekhar Panda, Manoranjan Nayak, Biswaranjan Das, Bikram Kumar Parida, Jayashree Jena, \\ Sukumar Bhakta, Sandeep Panda, Prasanna Kumar Panda, Lala Bihari Sukla*
}

Bioresources Engineering Department CSIR, Institute of Minerals and Materials Technology Bhubaneswar, 751013, Odisha, India

\begin{abstract}
A total of 150 algal samples were collected from 14 sampling sites of various fresh water and brackish water habitats of east coast region of Odisha, India during the period 2009-2011. About 41 algal strains were isolated and cultured into their pure forms. Out of the collected strains 5 taxa of Bacillariophyta, 12 taxa of Cyanobacteria/ Cyanoprokaryota and 24 taxa of Chlorophyta have been identified using relevant monographs and are being screend to evaluate their potential for biofuel production.
\end{abstract}

Keywords Algal diversity, Cyanobacteria, Bacillariophyta, Chlorophyta

\section{Introduction}

Odisha, located in the east coast of India (Lat. $17^{\circ} 48^{\prime}-23^{\circ}$ $34^{\prime} \mathrm{N} \&$ Long. $81^{\circ} 24^{\prime}-87^{\circ} 29^{\prime} \mathrm{E}$ ) has an area of $1,55,842$ $\mathrm{km}^{2}$ which is surrounded by West Bengal to the north-east, Jharkhand to the north, Chhattisgarh to the west and northwest and Andhra Pradesh to the south. In order to explore the algal biodiversity various field surveys was carried out during the year 2009-2011. Several water bodies which drain into the Bay of Bengal, provides a variety of ecological niches and a good habitats for the growth of algal species. Though there are several studies on algal biodiversity assessment in India have been carried out in the past[1-4], very comprehensive survey and seasonal collection of algal species in this typical location is few[5-7]. In the present study field trips were organized to carry out detail survey and collection of algal samples for screening and characterization of potential micro-algae for biodiesel production.

\section{Materials and Methods}

\subsection{Study Area}

Algal samples were collected randomly from 14 sampling sites of 8 estuaries of rivers and distributaries from coast of Odisha during 2009-2011 through several collection trips as shown in Table 1. In the southern part of the state, the estuaries of Rushikulya river along with its two major distributaries at Bahuda and Gopalpur were surveyed,

* Corresponding author:

suklalb@yahoo.co.in (Lala Bihari Sukla)

Published online at http://journal.sapub.org/env

Copyright (C) 2011 Scientific \& Academic Publishing. All Rights Reserved along with its distributaries viz., Kushabhadra, Nua nai, Devi along withits distributaries viz., Kushabhadra, Nua nai, Devi and Musa nai were visited for algal sample collection. Site map and different sampling sites for collection of micro algal samples are shown in Figure 1 and Figure 2.

\subsection{Sampling and Observation}

Samples were collected using forceps and needle and/or plankton net $(45 \mu \mathrm{m}$ pore size). Epilithic samples were scraped using a tooth brush. All algal samples were assigned voucher numbers and were preserved in a presterilized specimen bottle with $4 \%$ formaldehyde solution. Planktonic samples were fixed with Lugol's Iodine on the spot and brought to the laboratory for analysis. Simultaneously the replica of each sample was kept in the culture media and brought to the laboratory and incubated. Under microscope, single cells were isolated using glass capillary and put in the culture slants and incubated under light of 3000-4000 lux intensity. Cellular dimension measurement was carried out by micrometry and microphotograph of each specimen was taken using a Meiji ML-TH-05 Trinocular research microscope fitted with Nikon Coolpix 4500 digital camera. The organisms were identified using relevant monographs for various algal groups[8-11].

\subsubsection{Culture Conditions for Growing Microalgae}

- Temperature: All the cultures were maintained in an air conditioned culture room at a temperature of $25 \pm 2^{\circ} \mathrm{C}$.

- Intensity of Light: Light is the most vital factoring for the development of the photosynthetic algae both in in-vitro and in-vivo condition. The growth of algae is influenced by wave length, flux density and duration of light exposure. Hence the culture was maintained at light intensity 3000 4000 lux provided by cool white fluorescent tubes, with 
14:10 hr. of light / dark period.

- $\mathbf{p H}: \mathrm{pH}$ of cultures were maintained in the range of 6.87.0 .

- Growth Medium: The vital nutrients that enable the algae to grow through the culture medium are Macronutri- ents, Micronutrients and Agar-Agar (a solidifying agent).

The media used in the present study were Bold Basal for green algae, Walne and BG11 for cyanobacteria and modified BG11 for the diatom cultures, the details of which are presented in Table 2 .

Table 1. Details of collection from Odisha, East coast of India, in the year 2009-2011 showing place of collection, date of collection, habitat and voucher numbers

\begin{tabular}{|c|c|c|c|c|}
\hline Sl. no & Place of collection & $\begin{array}{c}\text { Date of } \\
\text { collection }\end{array}$ & Habitat & Voucher numbers \\
\hline 1 & Chilika lake, Balugaon, Khurda & 09.12 .2009 & $\begin{array}{l}\text { Planktonic, epilithic } \\
\text { (Temp. } 25^{\circ} \mathrm{C}, \mathrm{pH}-8.5 \text { ) }\end{array}$ & $1-4$ \\
\hline 2 & Temporary pool, Pathara, Ganjam & 09.12 .2009 & $\begin{array}{c}\text { Planktonic } \\
\left.\text { (Temp. } 33^{\circ} \mathrm{C}, \mathrm{pH}-8.7\right)\end{array}$ & 5 \\
\hline 3 & Palur canal, Ganjam & 09.12 .2009 & $\begin{array}{c}\text { Planktonic, floating } \\
\left(\text { Temp. } 29^{\circ} \mathrm{C}, \mathrm{pH}-8.9\right) \\
\end{array}$ & $6-9$ \\
\hline 4 & Bahuda estuary, Sonapur, Ganjam & 21.01.2010 & $\begin{array}{l}\text { Epilithic, planktonic, epizoic, filamentous } \\
\left(\text { Temp. } 26^{\circ} \mathrm{C}, \mathrm{pH}-8\right)\end{array}$ & $10-40$ \\
\hline 5 & Gopalpur estuary, Ganjam & 04.02 .2010 & $\begin{array}{l}\text { Planktonic, epizoic, benthic, filamentous } \\
\left.\text { (Temp. } 27^{\circ} \mathrm{C}, \mathrm{pH}-9\right)\end{array}$ & $41-47$ \\
\hline 6 & Rushikulya river, Gopalpur, Ganjam & 04.02 .2010 & $\begin{array}{l}\text { Benthic, epizoic, planktonic } \\
\left(\text { Temp. } 34^{\circ} \mathrm{C}, \mathrm{pH}-7.5\right)\end{array}$ & $48-55$ \\
\hline 7 & Rushikulya estuary, Ganjam & 04.02 .2010 & $\begin{array}{l}\text { Benthic, epilithic, floating } \\
\text { (Temp. } 28^{\circ} \mathrm{C}, \mathrm{pH}-8 \text { ) }\end{array}$ & $56-60$ \\
\hline 8 & Daya river, Bhubaneswar & 19.03.2010 & $\begin{array}{l}\text { Floating filamentous mat } \\
\text { (Temp. } 29^{\circ} \mathrm{C}, \mathrm{pH}-6.5 \text { ) }\end{array}$ & $61-62$ \\
\hline 9 & Kushabhadra estuary, Chandrabhaga, Puri & 25.03. 2010 & $\begin{array}{l}\text { Benthic, epilithic, epizoic, Planktonic, floating } \\
\text { (Temp. } 28^{\circ} \mathrm{C}, \mathrm{pH}-7.5 \text { ) }\end{array}$ & $63-74$ \\
\hline 10 & Nua nai estuary, Puri & 25. 03.2010 & $\begin{array}{l}\text { Benthic, epilithic, epizoic, Planktonic, floating } \\
\text { (Temp. } 32^{\circ} \mathrm{C}, \mathrm{pH}-8.5 \text { ) }\end{array}$ & $75-82$ \\
\hline 11 & Musa Nai estuary, Puri & 25. 03.2010 & $\begin{array}{l}\text { Benthic, epilithic, epizoic, Planktonic, floating } \\
\text { (Temp. } 33^{\circ} \mathrm{C}, \mathrm{pH}-7.5 \text { ) }\end{array}$ & $83-88$ \\
\hline 12 & Mahanadi river estuary, Paradeep & 16. 07.2010 & $\begin{array}{l}\text { Benthic, epilithic, epizoic, Planktonic, floating } \\
\left.\text { (Temp. } 33^{\circ} \mathrm{C}, \mathrm{pH}-7.5\right)\end{array}$ & $89-99$ \\
\hline 13 & $\begin{array}{l}\text { Subarnarekha estuary, Budhab alanga, } \\
\text { Kasafal, Dhamara }\end{array}$ & $3-6.01 .2011$ & $\begin{array}{l}\text { Benthic, epilithic, epizoic, Planktonic, filamentous } \\
\text { (Temp. } 18^{\circ} \mathrm{C}, \mathrm{pH}-6.8 \text { ) }\end{array}$ & $99-137$ \\
\hline 14 & Devi estuary, Astaranga & 07. 07. 2011 & $\begin{array}{l}\text { Benthic, epilithic, epizoic, Planktonic, floating } \\
\text { (Temp. } 27^{\circ} \mathrm{C}, \mathrm{pH}-6.7 \text { ) }\end{array}$ & $137-150$ \\
\hline \multicolumn{4}{|c|}{ Total number of vouchers collected } & 150 \\
\hline
\end{tabular}

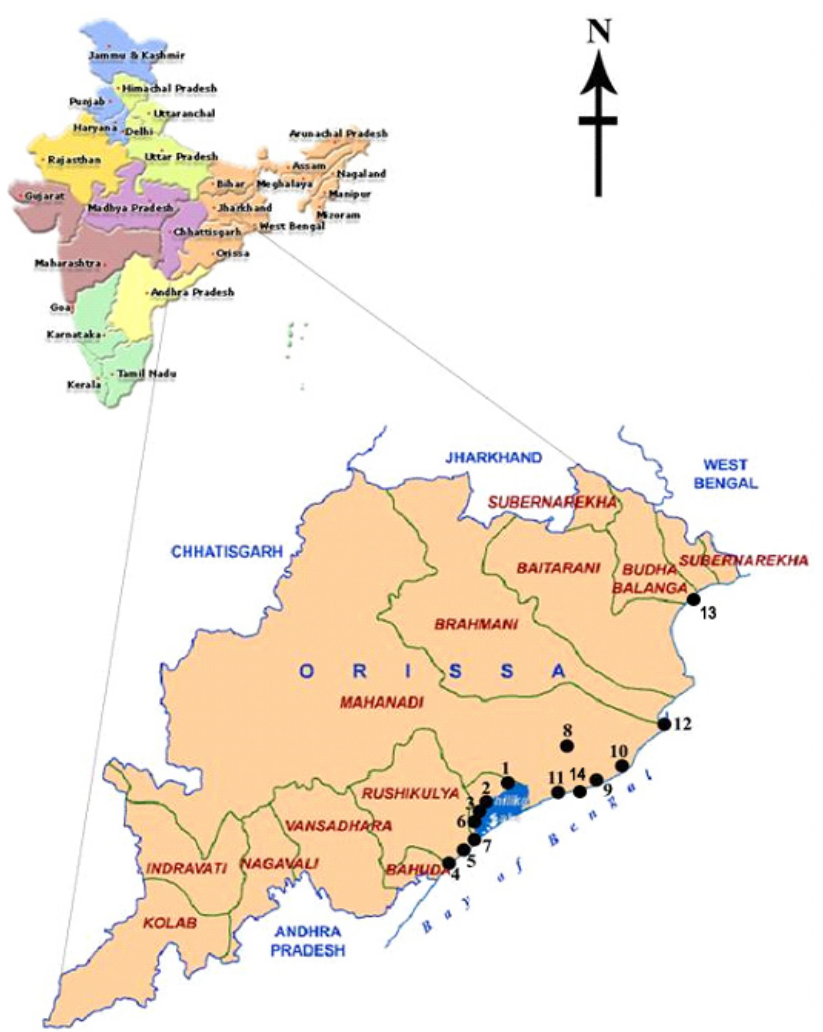

Figure 1. Site map of collection sites of Odisha. Black dots indicate the sampling sites

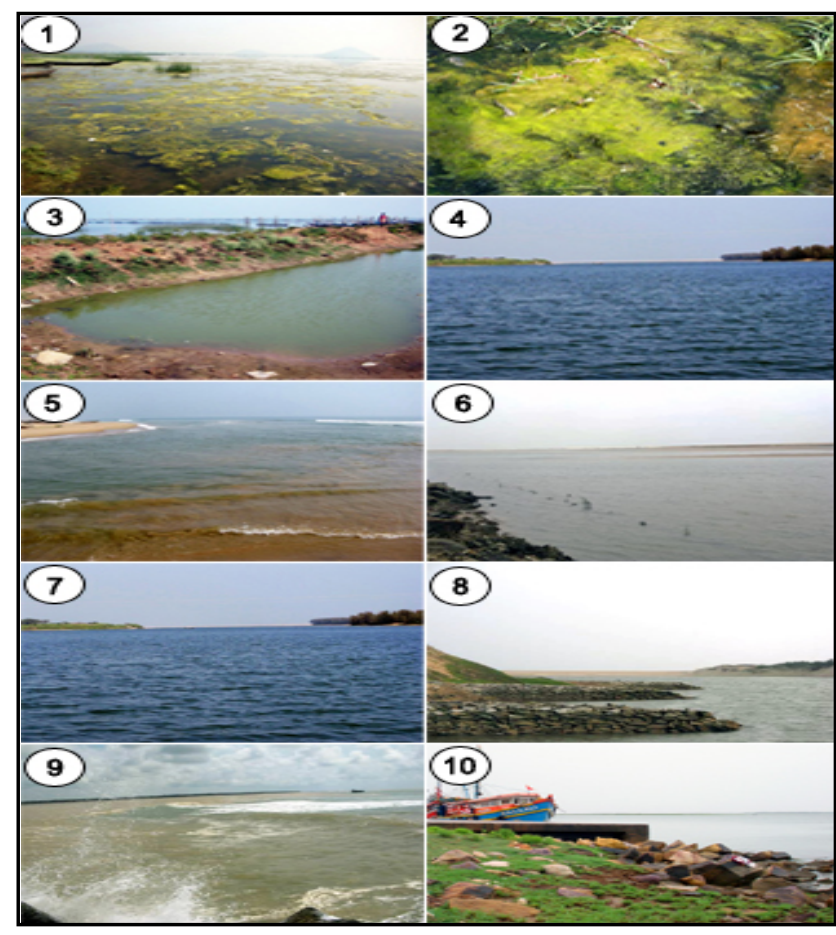

Figure 2. Respective photographs of collection sites of Odisha. 1. Chilika Lake, 2. Algal bloom in Chilika Lake, 3. Temporary Pool (Pathara), 4. Bahuda Estuary, 5. Rusikulya Estuary, 6.Kushabhadra estuary, 7. Nuanai river estuary, 8. Musa river estuary, 9. Mahanadi river estuary, 10. Devi estuary 
Table 2. Different growth medium were used for micro algal culture (a) Macronutrients (b) Micronutrients

\begin{tabular}{|c|c|c|c|}
\hline \multicolumn{4}{|c|}{ Macronutrients } \\
\hline Media composition & $\operatorname{BBM}(\mathrm{g} / \mathrm{l})$ & WALNE(g/l) & $\mathrm{BG}-11(\mathrm{~g} / \mathrm{l})$ \\
\hline $\mathrm{NaNO}_{3}$ & 0.25 & 0.1 & - \\
\hline $\mathrm{CaCl}_{2} \cdot 2 \mathrm{H}_{2} \mathrm{O}$ & 0.025 & - & - \\
\hline $\mathrm{MgSO}_{4} .7 \mathrm{H}_{2} \mathrm{O}$ & 0.075 & - & 0.075 \\
\hline $\mathrm{K}_{2} \mathrm{HPO}_{4}$ & 0.075 & - & 0.04 \\
\hline $\mathrm{KH}_{2} \mathrm{PO}_{4}$ & 0.175 & - & - \\
\hline $\mathrm{FeSO}_{4} .7 \mathrm{H}_{2} \mathrm{O}$ & 0.005 & - & - \\
\hline $\mathrm{Na}_{2}$ EDTA & 0.005 & 4.5 & 0.001 \\
\hline $\mathrm{NaCl}$ & 0.175 & - & - \\
\hline $\mathrm{MgCL}_{2 .} 6 \mathrm{H}_{2} \mathrm{O}$ & 2 & - & - \\
\hline $\mathrm{K}_{2} \mathrm{HPO}_{4} .3 \mathrm{H}_{2} \mathrm{O}$ & - & - & - \\
\hline $\mathrm{KCL}$ & - & - & - \\
\hline $\mathrm{Na}_{2} \mathrm{CO}_{3}$ & - & - & 0.02 \\
\hline Cirtic acid & - & - & 0.006 \\
\hline $\begin{array}{c}\text { Ferric Ammonium } \\
\text { Citrate }\end{array}$ & - & - & 0.006 \\
\hline MgEDTA & - & & - \\
\hline $\mathrm{NaH}_{2} \mathrm{PO}_{4} \cdot 2 \mathrm{H}_{2} \mathrm{O}$ & - & 0.02 & - \\
\hline $\mathrm{H}_{3} \mathrm{BO}_{3}$ & - & 0.0336 & - \\
\hline $\mathrm{MnCl}_{2} \cdot 4 \mathrm{H}_{2} \mathrm{O}$ & - & 3.6 & - \\
\hline $\mathrm{FeCl}_{3} \cdot 6 \mathrm{H}_{2} \mathrm{O}$ & - & 0.013 & - \\
\hline $\mathrm{NaSiO}_{3}$ & - & 6 & - \\
\hline \multicolumn{4}{|c|}{ Micronutrients } \\
\hline $\mathrm{H}_{3} \mathrm{BO}_{3}$ & 2.86 & - & 2.86 \\
\hline $\mathrm{MnCl}_{2} 4 \mathrm{H}_{2} \mathrm{O}$ & 1.81 & - & 1.81 \\
\hline $\mathrm{ZnSO}_{4} \cdot 7 \mathrm{H}_{2} \mathrm{O}$ & 0.222 & 0.044 & 0.222 \\
\hline $\mathrm{CuSO}_{4} .5 \mathrm{H}_{2} \mathrm{O}$ & 0.079 & 0.02 & 0.079 \\
\hline $\mathrm{Na}_{2} \mathrm{MoO}_{4} \cdot 2 \mathrm{H}_{2} \mathrm{O}$ & 0.390 & - & 0.390 \\
\hline $\mathrm{Co}\left(\mathrm{NO}_{3}\right)_{2} 6 \mathrm{H}_{2} \mathrm{O}$ & 0.0494 & - & 0.0494 \\
\hline $\mathrm{CoCl}_{2} \cdot 6 \mathrm{H}_{2} \mathrm{O}$ & - & 0.02 & - \\
\hline$\left(\mathrm{NH}_{4}\right) \mathrm{Mo}_{7} \mathrm{O}_{24} \mathrm{H}_{2} \mathrm{O}$ & - & 0.009 & - \\
\hline
\end{tabular}

\subsubsection{Modified BG11 for Diatom Culture}

The required nutrient composition for the Diatom culture was essentially same as that of the BG11 used for cyanobacterial culture, except for supplementing with double the concentration of $\mathrm{NO}_{3}{ }^{-}, \mathrm{PO}_{4}{ }^{3-}$ and $\mathrm{Na}_{2} \mathrm{SiO}_{3}$. Instead of distilled water, sea water was used for the Diatom culture.

\section{Results and Discussion}

\subsection{Isolation and Documentation of Algal Strain}

Forty one different algal strains were isolated and cultured into their pure forms and deposited in form of slants in the Culture Collection Centre \& Repository for brackish water algae at IMMT (CSIR), Bhubaneswar assigning with a strain number (Table 3 ). The microphotographs of the isolated algal samples are shown in Figure 3.

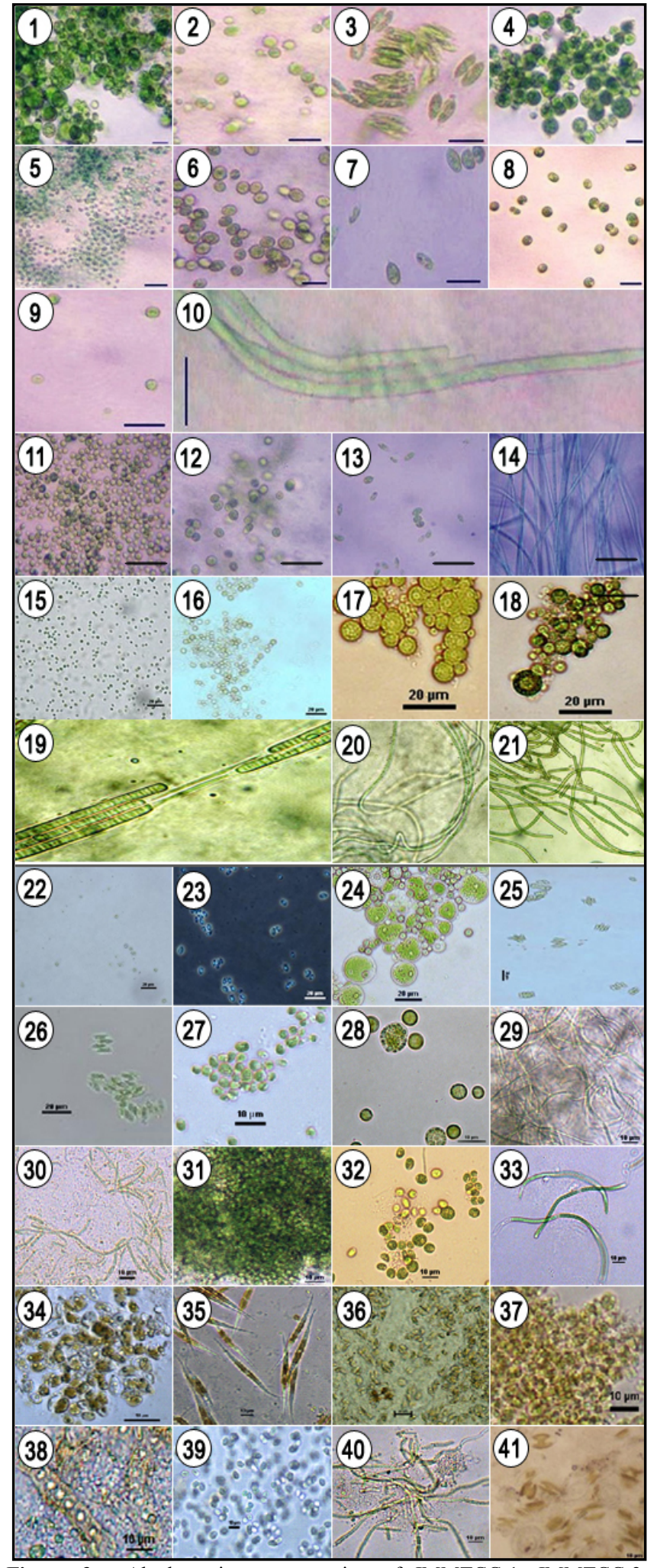

Figure 3. Algal strain representation of IMMTCC-1, IMMTCC-2, IMMTCC-3, IMMTCC-4, IMMTCC-5, IMMTCC-6, IMMTCC-7, IMMTCC-8, IMMTCC-9, IMMTCC-10, IMMTCC-11, IMMTCC-12, IMMTCC-13 and IMMTCC-14, IMMTCC-15, IMMTCC-16, IMMTCC17, IMMTCC-18, IMMTCC-19, IMMTCC-20, IMMTCC-21, IMMTCC22, IMMTCC-23, IMMTCC-24, IMMTCC-25, IMMTCC-26, IMMTCC27, IMMTCC-28, IMMTCC-29, IMMTCC-30, IMMTCC-31, IMMTCC32, IMMTCC-33, IMMTCC-34, IMMTCC-35, IMMTCC-36, IMMTCC37, IMMTCC-38, IMMTCC-39, IMMTCC-40, IMMTCC-41 
Table 3. List of algal strains maintained in IMMT Culture Collection Repository

\begin{tabular}{|c|c|c|}
\hline Sl.No. & No. of cultures collected so far & Strain Number \\
\hline 1 & Chlorococcum sp. & IMMTCC-01 \\
\hline 2 & Chlorella sp. & IMMTCC-02 \\
\hline 3 & Scenedesmus sp. & IMMTCC-03 \\
\hline 4 & Chlorococcum sp. & IMMTCC-04 \\
\hline 5 & Chlorella sp. & IMMTCC-05 \\
\hline 6 & Scenedesmus sp. & IMMTCC-06 \\
\hline 7 & Scenedesmus sp. & IMMTCC-07 \\
\hline 8 & Chlorella sp. & IMMTCC-08 \\
\hline 9 & Chlorella sp. & IMMTCC-09 \\
\hline 10 & Phormidium sp. & IMMTCC-10 \\
\hline 11 & Chlorococcum sp. & IMMTCC-11 \\
\hline 12 & Chlorococcum sp. & IMMTCC-12 \\
\hline 13 & Scenedesmus sp. & IMMTCC-13 \\
\hline 14 & Oscillatoria sp. & IMMTCC-14 \\
\hline 15 & Bracteococcus minor & IMMTCC-15 \\
\hline 16 & Chlorella sp. & IMMTCC-16 \\
\hline 17 & Chlorococcum sp. & IMMTCC-17 \\
\hline 18 & Chlorella sp. & IMMTCC-18 \\
\hline 19 & Phormidium sp. & IMMTCC-19 \\
\hline 20 & Phormidium sp. & IMMTCC-20 \\
\hline 21 & Oscillatoria sp. & IMMTCC-21 \\
\hline 22 & Oocystis sp. & IMMTCC-22 \\
\hline 23 & Chlamydomonas sp. & IMMTCC-23 \\
\hline 24 & Scenedesmus sp. & IMMTCC-24 \\
\hline 25 & Scenedesmus sp. & IMMTCC-25 \\
\hline 26 & Chlorococcum sp. & IMMTCC-26 \\
\hline 27 & Chlorella sp. & IMMTCC-27 \\
\hline 28 & Chroccodiopsis sp. & IMMTCC-28 \\
\hline 29 & Phormidium sp. & IMMTCC-29 \\
\hline 30 & Phormidium sp. & IMMTCC-30 \\
\hline 31 & Chlorella sp. & IMMTCC-31 \\
\hline 32 & Chlorella sp. & IMMTCC-32 \\
\hline 33 & Phormidium sp. & IMMTCC-33 \\
\hline 34 & Cymbella & IMMTCC-34 \\
\hline 35 & Nitzschia sp. & IMMTCC-35 \\
\hline 36 & Diatom & IMMTCC-36 \\
\hline 37 & Diatom & IMMTCC-37 \\
\hline 38 & Nostoc & IMMTCC-38 \\
\hline 39 & Aphanothece & IMMTCC-39 \\
\hline 40 & Nostoc & IMMTCC-40 \\
\hline 41 & Navicula & IMMTCC-41 \\
\hline
\end{tabular}

\section{Conclusions}

In the present field survey a total of 41 algal taxa have been identified from collection of 150 algal samples. Of these 12 taxa of Cyanobacteria/Cyanoprokaryota, 24 taxa of Chlorophyta and 5 taxa of Bacillariophyta are being and maintained in pure cultures. The collection of green algal species is being evaluated for their potentiality of biofuel production.

\section{ACKNOWLEDGEMENTS}

We thank Department of Biotechnology (DBT), Government of India for financial assistance and Prof. B.K. Mishra, Director, Institute of Minerals \& Materials Technology (CSIR) for providing laboratory facilities.

\section{REFERENCES}

[1] K.P. Biswas, "Common fresh and brackish water algal flora of India and Burma".Rec. Bot. Surv. India, vol. 15 (1): pp. 1105,1949

[2] I.Habib and U.K. Chaturvedi, "A systematic account of Chlorococcales from Ramnagar, Kumaun Himalaya". Phykos, vol. 38:pp. 97-100, 1999

[3] M. Jena, S.K. Ratha, and S.P. Adhikary, 2005 "Algal diversity changes in Kathjodi" river after receiving sewage of Cuttack and its ecological implication.vol. 8: pp. 67-74

[4] M. Jena, S.K. Ratha, and S.P. Adhikary, "Algal diversity of Rushikulya river, Orissa from origin till confluence to the sea”. Indian Hydrobiol., vol. 11 (1):pp. 9- 24, 2008

[5] M. Padhi, and S. Padhi, "Phytoplanktonic community of Gopalpur estuary". Seaweed Res. Utiln., vol. 21: pp. 95-97, 1999

[6] S.P. Adhikary, "A preliminary survey of algae of estuaries and coastal areas in Orissa". Seaweed Research and Utilization, 22:1-5, 2000

[7] S. Bhakta, S. K. Das, M. Nayak, J. Jena, P.K. Panda, L. B. Sukla, Phyco-Diversity Assessment of Bahuda River Mouth Areas of East Coast of Odisha, India, Recent Research in Science and Technology, vol. 2:pp.80-89, 2011

[8] T.V. Desikachary, Cyanophyta. 1959. I.C.A.R. monograph on Algae. New Delhi. pp. 686

[9] Philipose, M.T. 1967. Chlorococcales. I.C.A.R. Monographs on Algae, New Delhi, pp: 365

[10] Komárek, J. and Anagnostidis, K. 1998 Cyanoprokaryota1. Teil: Chroococcales. In: Ettl H, Gartner G, Heynig H and Mollenhauer D (eds.), SüßWasserflora. Von Mitteleuropa, Gaustav Fischer, vol. 19: pp 548

[11] Komárek, J. and Anagnostidis, K. 2005 Cyanoprokaryota11. Teil: Oscillatoriales. In: Büdel B, Gartner G, Krienitz L and Schagerl M (eds.), SüßWasserflora. Von Mitteleuropa, Elsevier, 19: Pp 759 